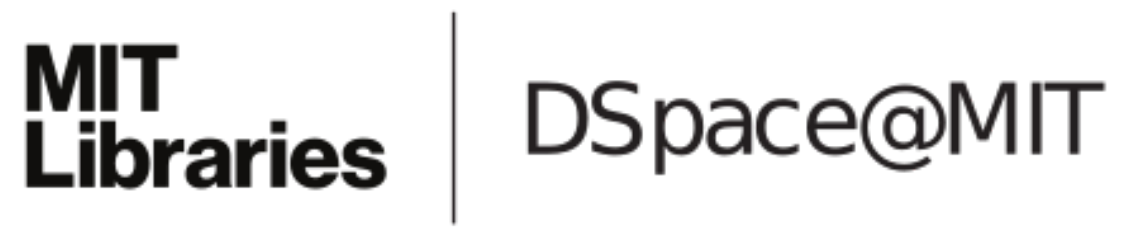

\author{
MIT Open Access Articles
}

High-throughput Microfluidics and Ultrafast
Optics for in Vivo Compound/Genetic Discoveries

The MIT Faculty has made this article openly available. Please share how this access benefits you. Your story matters.

Citation: Rohde, Christopher B. et al. "High-throughput microfluidics and ultrafast optics for in vivo compound/genetic discoveries." Imaging, Manipulation, and Analysis of Biomolecules, Cells, and Tissues VIII. Ed. Daniel L. Farkas, Dan V. Nicolau, \& Robert C. Leif. San Francisco, California, USA: SPIE, 2010. 75680N-6. (2010 COPYRIGHT SPIE--The International Society for Optical Engineering.

As Published: http://dx.doi.org/10.1117/12.841748

Publisher: SPIE

Persistent URL: http://hdl.handle.net/1721.1/58554

Version: Final published version: final published article, as it appeared in a journal, conference proceedings, or other formally published context

Terms of Use: Article is made available in accordance with the publisher's policy and may be subject to US copyright law. Please refer to the publisher's site for terms of use. 


\title{
High-throughput microfluidics and ultrafast optics for in vivo compound/genetic discoveries
}

\author{
Christopher B. Rohde, Cody Gilleland, Chrysanthi Samara, M. Fatih Yanik* \\ Massachusetts Institute of Technology, 77 Massachusetts Ave., Cambridge, MA, USA 02139-4307 \\ *yanik@mit.edu
}

\begin{abstract}
Therapeutic treatment of spinal cord injuries, brain trauma, stroke, and neurodegenerative diseases will greatly benefit from the discovery of compounds that enhance neuronal regeneration following injury. We previously demonstrated the use of femtosecond laser microsurgery to induce precise and reproducible neural injury in C. elegans, and have developed microfluidic on-chip technologies that allow automated and rapid manipulation, orientation, and non-invasive immobilization of animals for sub-cellular resolution two-photon imaging and femtosecond-laser nanosurgery. These technologies include microfluidic whole-animal sorters, as well as integrated chips containing multiple addressable incubation chambers for exposure of individual animals to compounds and sub-cellular time-lapse imaging of hundreds of animals on a single chip. Our technologies can be used for a variety of highly sophisticated in vivo high-throughput compound and genetic screens, and we performed the first in vivo screen in C. elegans for compounds enhancing neuronal regrowth following femtosecond microsurgery. The compounds identified interact with a wide variety of cellular targets, such as cytoskeletal components, vesicle trafficking, and protein kinases that enhance neuronal regeneration.
\end{abstract}

Keywords: Femtosecond, laser surgery, neural regeneration, C. elegans, microfluidics, high-throughput.

\section{INTRODUCTION}

The size and complexity of the instrumentation used to study large vertebrate animal models prohibits their use in highthroughput assays for rapid identification of new genes and drug targets. Because of this, researchers turn to simpler organisms, and the advantages of using small invertebrate animals as model systems for human disease have become increasingly apparent. The nematode Caenorhabditis elegans (C. elegans) is a powerful model organism due to a number of useful properties including its small size, optical transparency, rapid developmental cycle and the availability of a wide array of species-specific genetic techniques. However, since the first studies on C. elegans in the early 1960s, little has changed in how scientists manipulate this tiny organism by manually picking, sorting, and transferring individual animals. The reliance on manual techniques means that large-scale forward- and reverse-genetic screens can take several months to years to complete. The high-throughput techniques that exist in C. elegans require assays to be significantly simplified in order to be even partially automated.

Fortunately, many of the properties that make C. elegans a useful model organism also make it well suited to manipulation in microfluidic channels. This has enabled us to create components for an integrated, whole-animal, highthroughput sorting and large-scale screening platform to perform drug and genetic assays with sub-cellular resolution. We have designed microfluidic devices [1][2] from multiple layers of the flexible elastomer poly(dimethyl siloxane) [3] that can be combined in various configurations to allow a multitude of complex high-throughput assays such as mutagenesis, drug and RNAi screens. These devices are: (i) a small-animal sorter that enables highly-stable of immobilization live, unanesthetized animals; (ii) an array of microfluidic chambers for simultaneous incubation, immobilization, sub-celluar-resolution imaging and independent screening of many animals on a single chip; and (iii) a microfluidic interface to large-scale multiwell-format libraries that also functions as a multiplexed animal dispenser.

The rapid and repeatable animal immobilization which the devices enable allows high-throughput and precise surgery. Following surgery, animals are exposed to the contents of a small-molecule library and assayed for neuronal regeneration. Using this screening method we have identified several compounds that appear to enhance neural regeneration in vivo.

Imaging, Manipulation, and Analysis of Biomolecules, Cells, and Tissues VIII, edited by Daniel L. Farkas, Dan V. Nicolau, Robert C. Leif, Proc. of SPIE Vol. 7568, 75680N · C 2010 SPIE · CCC code: 1605-7422/10/\$18 - doi: 10.1117/12.841748 


\section{ON-CHIP HIGH-THROUGHPUT IMMOBILIZATION AND SORTING}

The high mobility of $C$. elegans requires them to be immobilized in order to examine cellular and sub-cellular features. This is most commonly done using anesthesia such as sodium azide $\left(\mathrm{NaN}_{3}\right)$, levamisole, or tricaine/tetrimisole. However, anesthetics can have negative or uncharacterized side-effects on biological processes. Cooling can also be used to reversibly immobilize animals, however this can also have unpredictable effects, especially since many $C$. elegans strains are especially sensitive to temperature variations. Additionally, these immobilization techniques are not suitable for assays requiring physiologically active animals, such as investigations into germ-line proliferation, development, or neurophysiology.

We have developed a microfluidic small-animal sorter that can rapidly isolate and immobilize individual animals. The sorter consists of control channels and valves (gray) that direct the flow of worms in the flow channels in different directions (Figure 1) [1]. A single animal is captured using the suction point located at the top side of the channel. During this time the lower suction channels are inactive. To isolate this animal, the chamber is then washed to flush any other worms in the chamber (blue line) toward the waste or back towards the circulating input. The chamber is isolated from all of the channels and the worm is released from the top suction channel. The worm is then restrained by the lower suction channels (red line). This immobilizes animals only partially, and is not sufficient to completely restrict their motion. In order to fully immobilize the animals, we create a seal around them that restricts their motion completely. using a 15-25 $\mu \mathrm{m}$-thick flexible sealing membrane that separates a press-down channel from the flow channel below (Figure 1e) [2]. The press-down channel can be rapidly pressurized to expand the thin membrane downwards, wrapping around the animals and forming a tight seal which completely constrains their motion in a linear orientation. Following immobilization, the worm can be imaged at sub-cellular resolution using high-resolution optics. The stability achieved is sufficient to allow three-dimensional imaging and sub-cellular manipulation using femotosecond laser micro-surgery (see below). Following phenotype identification the captured animal is released and can be directed to one of two collection channels. Quantitative analysis of the immobilization stability shows that it is comparable to chemical anesthetics, and there was no change in the lifespan or brood size of the immobilized animals [2]. Additionally, visual observation of the animals and their neurons showed no signs of hypoxia or other distress. 

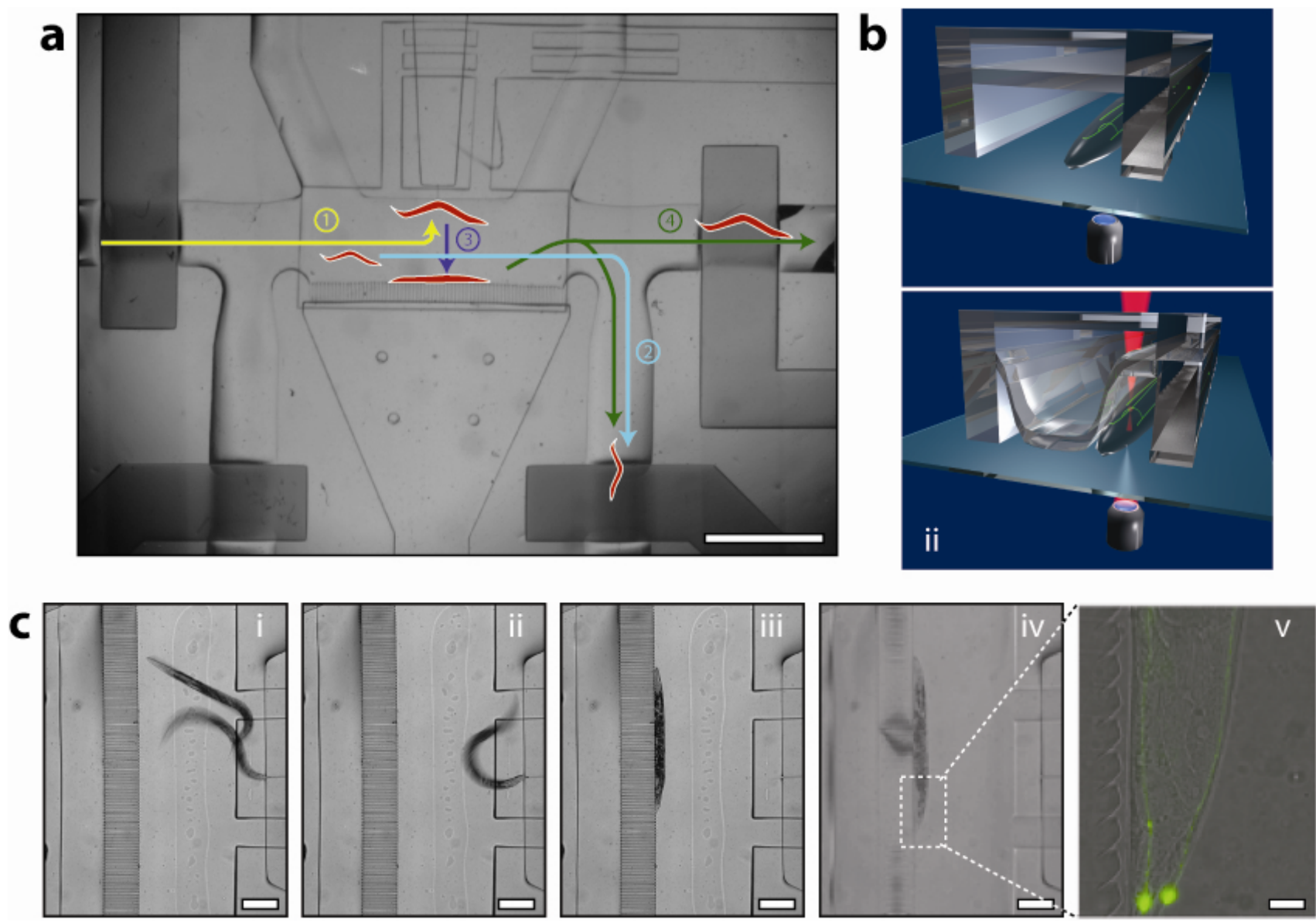

Figure 1. Microfluidic immobilization for imaging and femtosecond laser microsurgery of C. elegans. (a) Micrograph of chip with numbered arrows showing microfluidic C. elegans manipulation steps. 1: Loading of nematodes and capture of a single animal by one aspiration channel. 2: Washing of the channels to remove and recycle the rest of the nematodes. 3: Release of the captured animal from single aspiration port, and recapture and orientation of it by a linear array of aspiration ports. 4: Collection of the animal post surgery. Scale bar: $1 \mathrm{~mm}$. (b) Illustration of the final immobilization and laser axotomy: Once a single animal is captured and linearly oriented (i), a channel above it is pressurized pushing a thin membrane downwards (ii). This membrane wraps around the animal significantly increasing immobilization stability for imaging and surgery. (c) Single animal capture (i), isolation (ii), linear orientation (iii), and compressive immobilization (iv), superposition of bright-field image of an animal and its pmec4::gfp fluorescent labeled lateral mechanosensory (PLM) neurons (v). Scale bars: (i)-(iv) $250 \mu \mathrm{m}$, (v) $20 \mu \mathrm{m}$.

\section{LARGE-SCALE TIME-LAPSE STUDIES USING MULTIPLEXED INCUBATION CHAMBERS}

High-throughput time-lapse studies on small animals are currently performed in multiwell plates by automated fluorescence microplate readers [4]. Because the animals swim inside the wells, only average fluorescence is obtained from each well, and cellular and sub-celluar details cannot be imaged. Although anesthesia can be used to immobilize the animals, the effects of long-term anesthesia prohibit many time-lapse screens, and anesthesia can have detrimental effects on many biological processes. Additionally, animal loss can occur during media exchange. To address these problems, we designed the microfluidic-chamber device shown in Figure 2(a) for worm incubation and for continuous imaging at sub-celluar resolution. Sorted worms can be delivered to the chambers by opening valves via multiplexed control lines [5].

Microchamber chips based on this design can be readily scaled for large-scale screening applications because the number of control lines required to independently address $n$ incubation chambers scales only with $\log (n)$ [5]. The 
millimeter scale of the microchambers can allow hundreds of microchambers to be integrated on a single chip. To image animals, a flow is used to push the animals toward the posts arranged in an arc inside the chambers (Figures 2(b) and 2(c)). This flow restrains the animals for sub-celluar imaging. The arrangement of the posts positions animals in a similar configuration, which simplifies the analysis of images. The medium in the chambers can be exchanged through the microfluidic channels for complex screening strategies, and precisely timed exposures to biochemicals (e.g., drugs/RNAi) can be performed. The use of microfluidic technology also reduces the cost of whole-animal assays by reducing the required volumes of compounds.
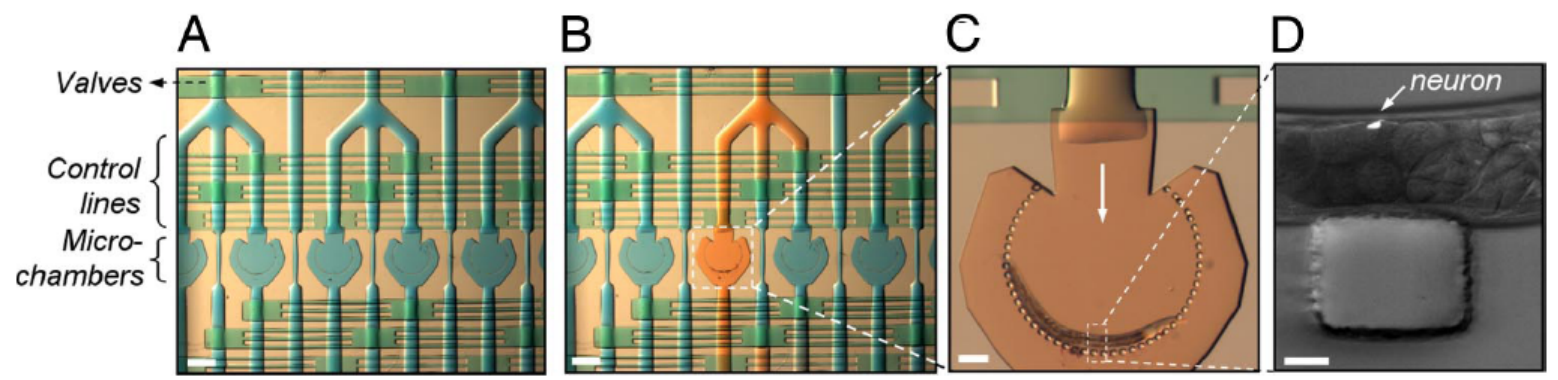

Figure 2. Individually addressable microfluidic screening chambers. a) Device layout illustrating flow (blue) and control (green) lines. The center channel between the two chambers can be used to wash the lines leading up to the individual chambers. (b) Illustration of individual chamber addressing. (c) Semi-circular post arrangement allows animal immobilization using a controlled flow. Once immobilized, animals can be imaged inside the chambers. (d) High-resolution image taken through glass substrate (scale bars a-b: $500 \mu \mathrm{m}$, c: $100 \mu \mathrm{m}$, d: $25 \mu \mathrm{m}$ ).

\section{MULTI-WELL PLATE INTERFACING FOR LOADING COMPOUNDS}

Interfacing microfluidics to existing large-scale RNAi and drug libraries in standard multiwell plates represents a significant challenge as it is impractical to deliver compounds to hundreds of microchambers on a single chip through hundreds of external fluidic connectors. The interface device in Figure 3 addresses this problem. This microfluidic interface chip consists of an array of aspiration tips that can be lowered into the wells of microwell plates, which allows minute amounts of library compounds to be collected from the wells by suction, routed through multiplexed flow lines one at a time, and delivered to the single output of the device. Following delivery the lines of the chip can be easily and automatically washed to prevent cross-contamination. The output of the interface chip can then be connected to our microfluidic-chamber device for sequential delivery of compounds to each microchamber. Combining this multiwellplate interface chip with existing robotic multiwell-plate handlers will allow large libraries to be delivered to microfluidic chips. The same device can also be used to dispense worms into multiwell plates, simply by running it in reverse.

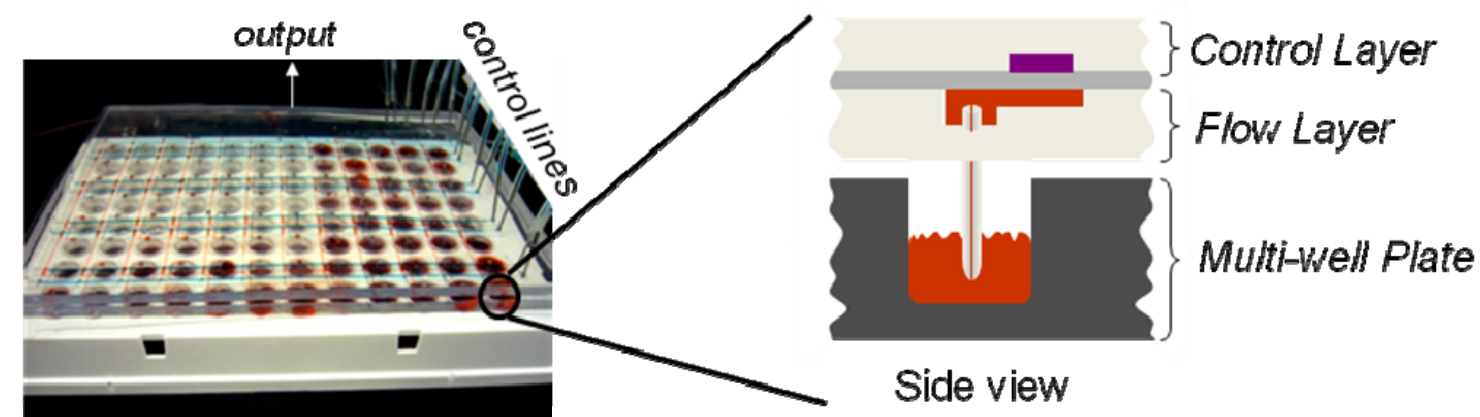

Figure 3: Microfluidic interface device for large-scale compound delivery. Control lines allow addressing of individual wells via suction through pins seated on a multiwell plate. When connected to the output of a microfluidic chip, the same flow lines can be used to dispense individual animals into wells. 


\section{HIGH-THROUGHPUT ON-CHIP FEMTOSECOND LASER MICROSURGERY}

Femtosecond-laser micro/nanosurgery enables precise ablation of sub-cellular processes with minimal collateral damage [6] and we have previously employed this technique to perform the first axonal regeneration study in C. elegans [7][8]. However, manually preparing an animal for surgery, imaging and recovering it afterwards are laborious. Additionally, the effects of long-term anesthesia on biological processes are not known. We can use our immobilization technique to repeatably and rapidly immobilize animals and perform femtosecond-laser microsurgery with sub-cellular precision.

Another application requiring an even higher degree of stabilization is multi-photon microscopy [9]. This technique has the ability to perform optical sectioning with negligible out-of-plane absorption and emission due to its non-linearity. This dramatically reduces photobleaching and phototoxicity, [10] which is especially significant in assays that require animals to be imaged at multiple time points. Both of these applications are illustrated in Fig 4.

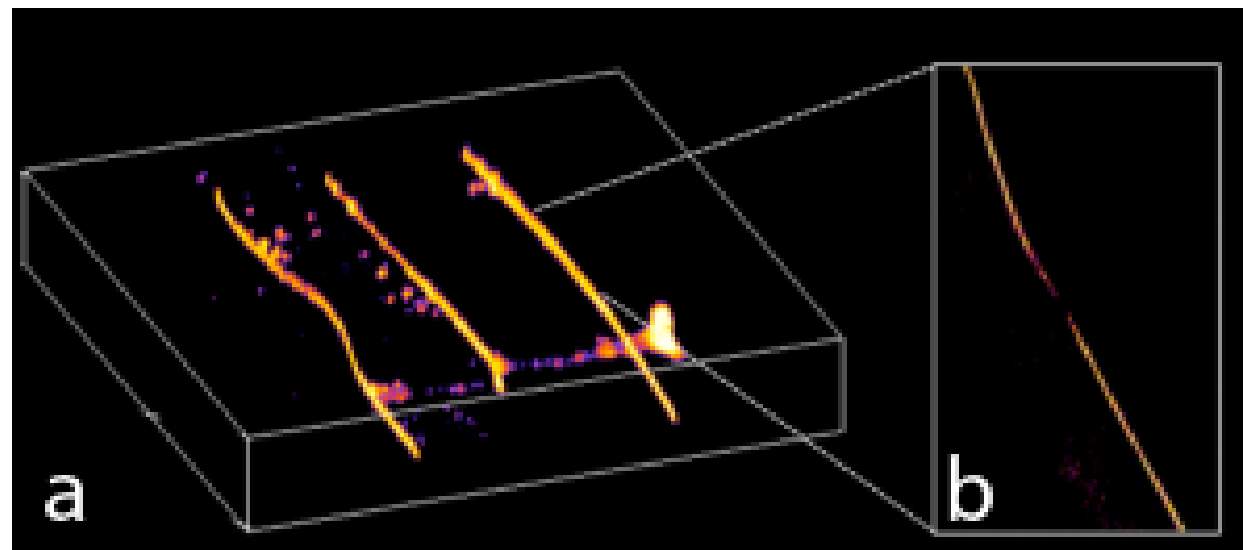

Figure 4. On-chip small-animal immobilization allows the use of several optical techniques. (a) Three-dimensional two-photon imaging and (b) femtosecond laser microsurgery can be performed on awake animals. (a) shows the volume reconstruction of the mid-body axons visible in a pmec4::gfp animal. The image was obtained with twophoton microscopy using a $40 \times / 0.8$ NA water-immersion objective. A slow scan rate is used to demonstrate the stability of animal immobilization by imaging a $120 \mu \mathrm{m} \times 120 \mu \mathrm{m} \times 30 \mu \mathrm{m}$ volume in two minutes. Femtosecond laser microsurgery was performed using a Mai-Tai ${ }^{\circledR}$ HP (Spectra-Physics) femtosecond laser beam with $800 \mathrm{~nm}$ wavelength and $80 \mathrm{MHz}$ repetition rate. The cutting beam was delivered to a 20x/0.75 NA objective lens inside a Nikon TE2000 microscope. Axons were cut by $5 \mathrm{~nJ}$ pulses lasting $3.2 \mathrm{~ms}$.

\section{SCREENING FOR FACTORS AFFECTING NEURAL REGENERATION}

The speed and accuracy of immobilization achieved by our microfluidic devices enables large-scale screening of chemical and genetic libraries. We have used these devices to screen for compounds enhancing neural regeneration. Following axotomy by femtosecond laser microsurgery, animals were subsequently exposed to elements of a smallmolecule library containing a wide variety of compounds. By analyzing the length of regenerating axons following compound exposure, we identified several compounds that enhance neuronal regeneration in vivo (Figure 5). By performing various RNAi screens using femtosecond laser microsurgery and microfluidic technologies, we also identified some of the genetic targets of these compounds. 

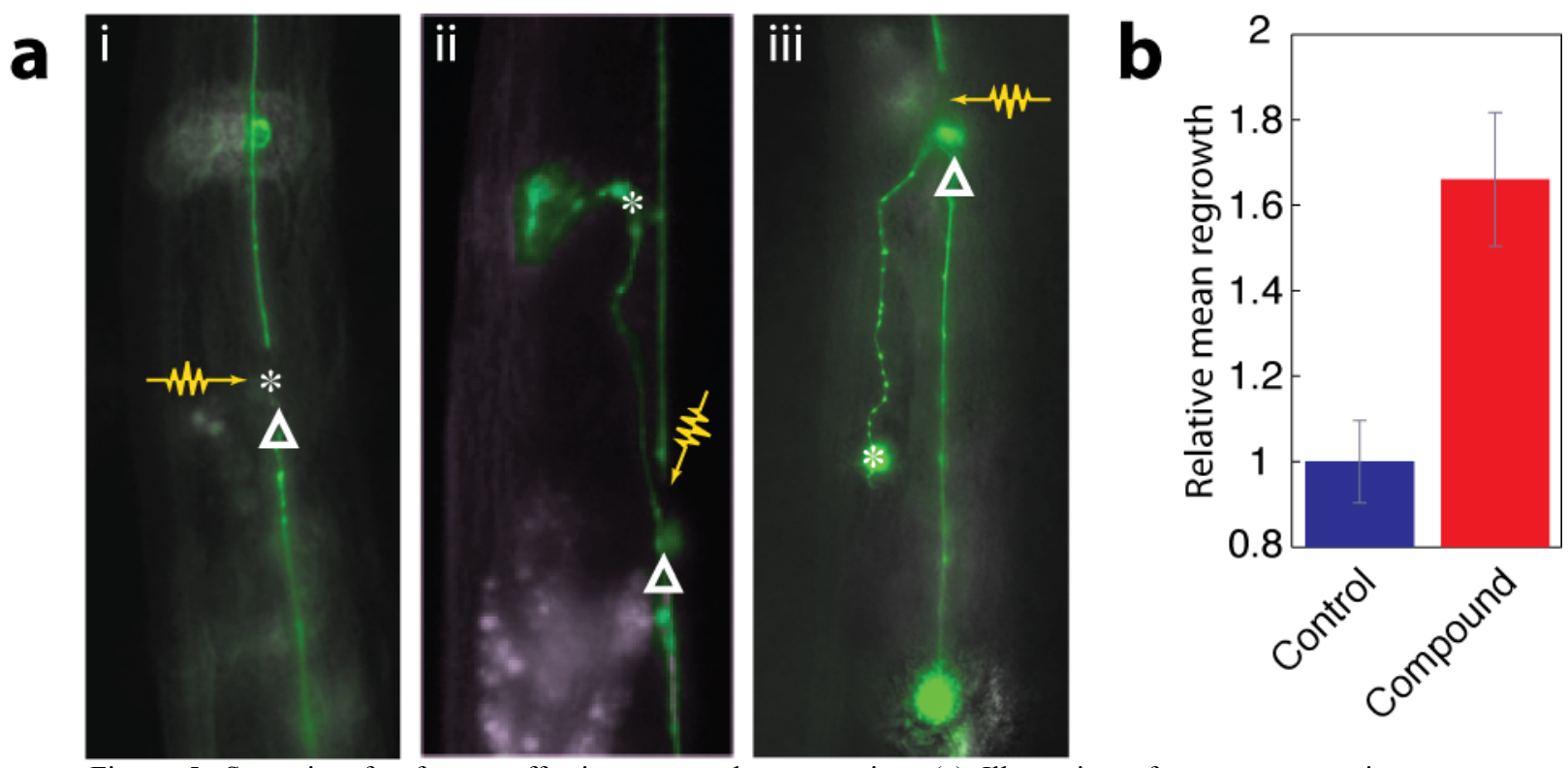

Figure 5. Screening for factors affecting neuronal regeneration. (a) Illustration of some regeneration types observed following femtosecond laser microsurgery and compound exposure. Arrows indicate original surgery location, triangles $(\Delta)$ and asterisks $(*)$ indicate start and end points of regenerated regions, respectively. (b) Enhancement following compound exposure.

\section{REFERENCES}

[1] Rohde, C.B., Zeng, F., Gonzalez-Rubio, R., Angel, M., and Yanik, M.F., "Microfluidic system for on-chip highthroughput whole-animal sorting and screening at subcellular resolution," Proc Natl Acad Sci U S A 104, 1389113895 (2007).

[2] Zeng, F., Rohde, C.B., and Yanik, M.F., "Sub-cellular precision on-chip small-animal immobilization, multi-photon imaging and femtosecond-laser manipulation," Lab Chip 8, 653-656 (2008).

[3] Kaletta, T., Butler, L., and Bogaert, T., Model organisms in drug discovery (2003).

[4] Xia, Y.N., and Whitesides, G.M., "Soft lithography.," Abstracts of Papers of the American Chemical Society 214, 348-PMSE (1997).

[5] Melin, J., and Quake, S.R., "Microfluidic large-scale integration: the evolution of design rules for biological automation," Annu Rev Biophys Biomol Struct 36, 213-231 (2007).

[6] Vogel, A., Noack, J., Huttman, G., and Paltauf, G., "Mechanisms of femtosecond laser nanosurgery of cells and tissues," Applied Physics B-Lasers and Optics 81, 1015-1047 (2005).

[7] Yanik, M.F., Cinar, H., Cinar, H.N., Chisholm, A.D., Jin, Y., and Ben-Yakar, A., "Neurosurgery: functional regeneration after laser axotomy," Nature 432, 822 (2004).

[8] Yanik, M.F., Cinar, H., Cinar, H.N., Gibby, A., Chisholm, A.D., Jin, Y.S., and Ben-Yakar, A., "Nerve regeneration in Caenorhabditis elegans after femtosecond laser axotomy," Ieee Journal of Selected Topics in Quantum Electronics 12, 1283-1291 (2006).

[9] Denk, W., Strickler, J.H., and Webb, W.W., "Two-photon laser scanning fluorescence microscopy," Science 248, 73-76 (1990).

[10]Filippidis, G., Kouloumentas, C., Voglis, G., Zacharopoulou, F., Papazoglou, T.G., and Tavernarakis, N., "Imaging of Caenorhabditis elegans neurons by second-harmonic generation and two-photon excitation fluorescence," J Biomed Opt 10, 024015 (2005). 